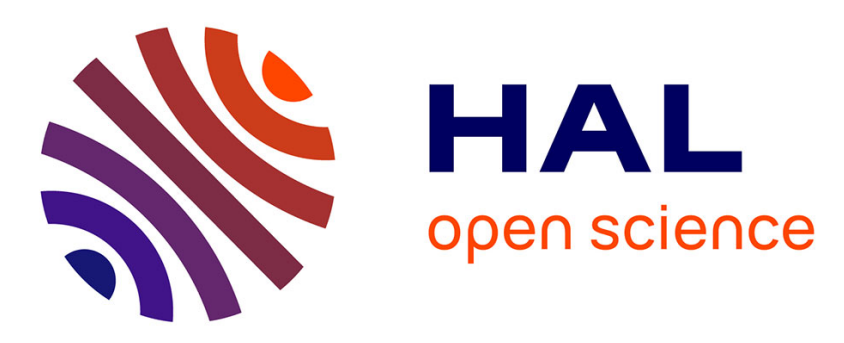

\title{
Generation of Learning Samples for Historical Handwriting Recognition Using Image Degradation
} Andreas Fischer, Muriel Visani, van Cuong Kieu, Ching Y. Suen

\section{To cite this version:}

Andreas Fischer, Muriel Visani, van Cuong Kieu, Ching Y. Suen. Generation of Learning Samples for Historical Handwriting Recognition Using Image Degradation. 2nd International Workshop on Historical Document Imaging and Processing, Aug 2013, Washington, DC, USA, United States. hal01006088

\section{HAL Id: hal-01006088 \\ https://hal.science/hal-01006088}

Submitted on 13 Jun 2014

HAL is a multi-disciplinary open access archive for the deposit and dissemination of scientific research documents, whether they are published or not. The documents may come from teaching and research institutions in France or abroad, or from public or private research centers.
L'archive ouverte pluridisciplinaire HAL, est destinée au dépôt et à la diffusion de documents scientifiques de niveau recherche, publiés ou non, émanant des établissements d'enseignement et de recherche français ou étrangers, des laboratoires publics ou privés. 


\section{Generation of Learning Samples for Historical Handwriting Recognition Using Image Degradation}

\author{
Andreas Fischer \\ CENPARMI, Concordia University \\ Montreal, Canada \\ an_fisch@encs.concordia.ca
}

\author{
Muriel Visani \\ L3i, University of La Rochelle \\ La Rochelle, France \\ muriel.visani@univ-Ir.fr
}

\author{
Kieu Van Cuong \\ LaBRI, University of Bordeaux I \\ Bordeaux, France \\ vkieu@labri.fr \\ Ching Y. Suen \\ CENPARMI, Concordia University \\ Montreal, Canada \\ suen@encs.concordia.ca
}

\begin{abstract}
Historical documents pose challenging problems for training handwriting recognition systems. Besides the high variability of character shapes inherent to all handwriting, the image quality can also differ greatly, for instance due to faded ink, ink bleed-through, wrinkled and stained parchment. Especially when only few learning samples are available, it is difficult to incorporate this variability in the morphological character models. In this paper, we investigate the use of image degradation to generate synthetic learning samples for historical handwriting recognition. With respect to three image degradation models, we report significant improvements in accuracy for recognition with hidden Markov models on the medieval Saint Gall and Parzival data sets.
\end{abstract}

\section{Keywords}

Historical Documents, Handwriting Recognition, Learning Sample Generation, Synthetic Images, Image Degradation, Hidden Markov Models

\section{INTRODUCTION}

The interest in handwriting recognition for historical documents has been growing rapidly in recent years [1]. Automatic reading of historical manuscripts would provide access to the contents of a vast amount of digitized historical documents stored at libraries worldwide. However, current handwriting recognition systems are still far from being perfect. Typical problems include the high variability in character shapes, the large number of word classes, and the inability to segment touching and broken characters prior to recognition [12]. For historical documents, an additional problem are differences in the image quality stemming for instance from faded ink, ink bleed-through, wrinkled and stained parchment. While this problem has been mainly addressed in the context of layout analysis [14], it has also an effect on learning-based recognition systems. Indeed, the morphological character models have to incorporate different degradations at learning stage in order to generalize well to unseen test images.

Image degradation is a promising approach to distort available learning samples in order to enlarge the training set and incorporate more variability in the image quality. Diverse image degradation models have been proposed in document analysis [2], for example barcode printing defects [15], ink bleed-through [17], and "hard pencil noise" [7]. A typical application scenario is the evaluation of document analysis systems under different degrees of distortion, for instance to assess symbol recognition and spotting [3], music score staff removal [22], and handwriting recognition [18].

In our application scenario, image degradation is used to generate learning samples. This approach has been pursued in $[20,21]$ for modern handwriting. Several perturbation models were applied to the handwriting images to distort the shape of the characters and the baseline of the text lines. Promising improvements of the recognition accuracy are reported with synthetic learning samples. However, aiming at modern handwritings, this degradation model does not provide the means to generate typical noise observed in digitized historical manuscripts.

In this paper, we investigate the case of historical handwriting based on three degradation models, namely Kanungo noise [9], a character degradation model [10], and a geometric distortion model [13]. They are applied to binary text line images to generate additional learning samples for hidden Markov model based recognition. On the medieval Saint Gall [4] and Parzival [6] data sets, we demonstrate that the recognition accuracy can be significantly improved with the synthetic learning samples.

The remainder of this paper is organized as follows. First, the data sets are introduced in Section 2. Next, the image degradation models are detailed in Section 3. Section 4 provides information about the recognition system and Section 5 presents the experimental results. Finally, we draw some conclusions in Section 6. 
Table 1: Data Sets

\begin{tabular}{lcc}
\hline & Saint Gall & Parzival \\
\hline Century & 9 th & 13 th \\
Language & Latin & German \\
Writers & 1 & 3 \\
Pages & 60 & 47 \\
Text Lines & 1,410 & 4,477 \\
\hline
\end{tabular}

\section{DATA SETS}

Two medieval data sets of the publicly available IAM-HistDB ${ }^{1}$ are used in this paper, namely the Saint Gall database and the Parzival database. Data set statistics are listed in Table 1 and sample images are shown in Figures 1a and $1 \mathrm{~b}$. The images illustrate considerable differences in the image quality even within a single manuscript.

\subsection{Saint Gall Database}

The Saint Gall database is based on a medieval Latin manuscript from the 9th century that contains the hagiography Vita sancti Galli by Walafrid Strabo. The Abbey Library of Saint Gall, Switzerland, holds a manual copy of the work within the Cod. Sang. 562, which was written by a (probably) single experienced hand in Carolingian script with ink on parchment.

The database currently includes 60 manuscript pages as indicated in Table 1. For further details on this database, we refer to [4].

\subsection{Parzival Database}

The Parzival database is based on a medieval German manuscript from the 13th century that contains the epic poem Parzival by Wolfram von Eschenbach, one of the most significant epics of the European Middle Ages. There exist several manual copies of the poem that differ in writing style and dialect of the language. For the Parzival database, the Cod. 857 is considered, which is held by the Abbey Library of Saint Gall, Switzerland. It was written in Middle High German by several writers with ink on parchment using Gothic minuscules.

The Parzival database currently consists of 47 manuscript pages as indicated in Table 1. For further details on this database, we refer to [6].

\section{IMAGE DEGRADATION MODELS}

In this section, the three image degradation models are presented that are used to generate synthetic learning samples. They are applied to binary text line images after a series of image preprocessing steps.

The resulting degradation images have been made publicly available. $^{2}$

\footnotetext{
${ }^{1}$ http://www.iam.unibe.ch/fki/databases/

iam-historical-document-database

${ }^{2}$ http://www.labri.fr/perso/vkieu/content/

Databases/hwr_parzival_saintgal.html
}
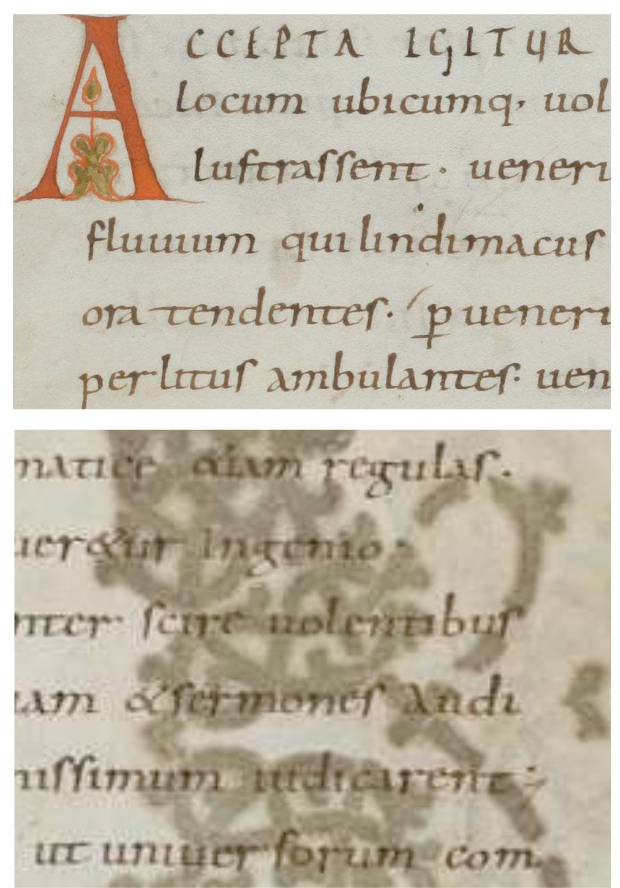

(a) Saint Gall Database

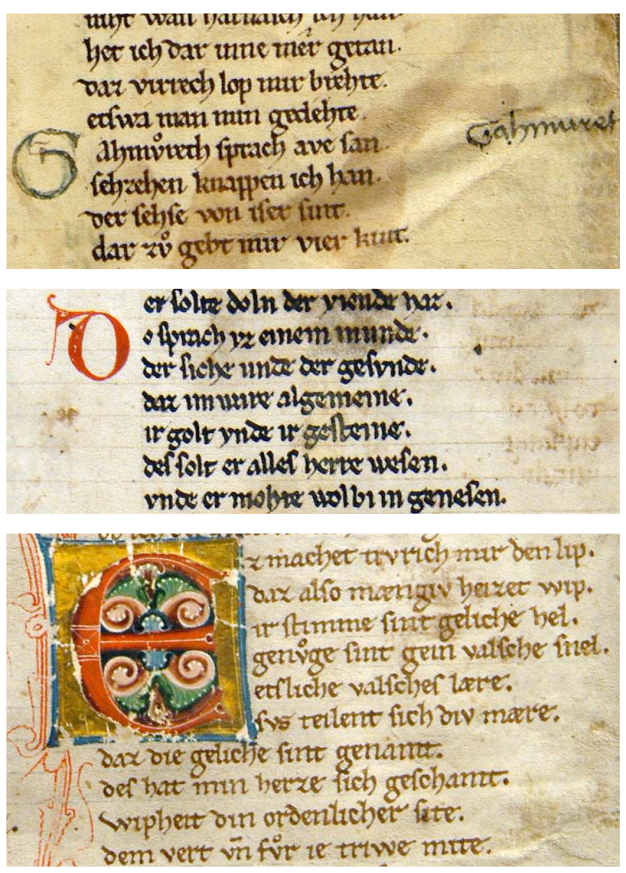

(b) Parzival Database

Figure 1: Data Sets 


\section{ute nomen eur bragantum:}

(a) Original

\section{ur nomen euur brgantum;}

(b) Normalization

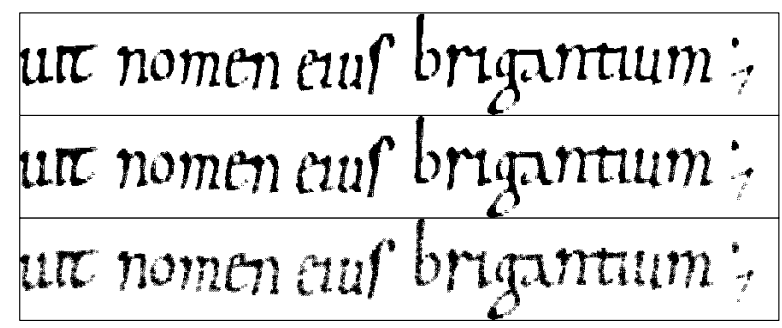

(c) Kanungo

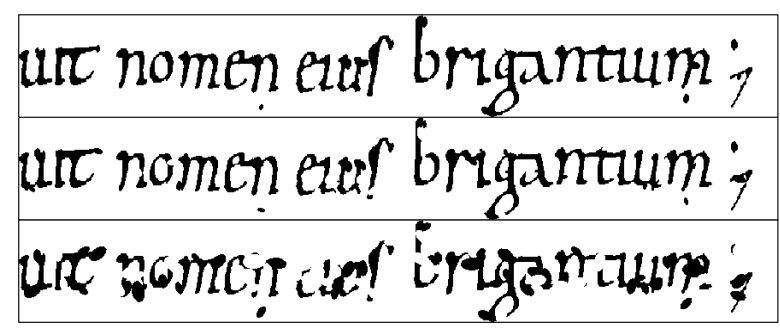

(d) Character

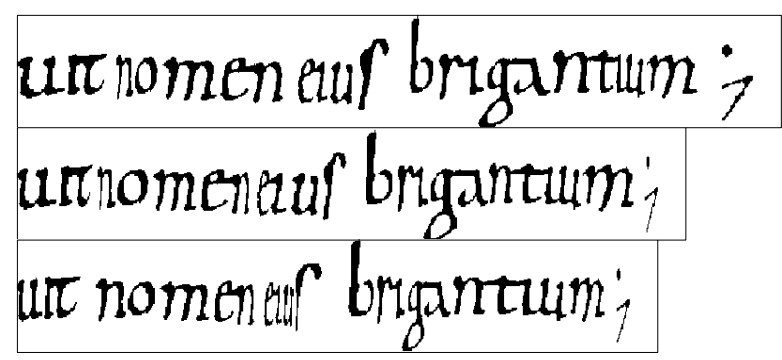

(e) Geometric

Figure 2: Image Degradation Saint Gall

\subsection{Preprocessing}

The first step in preprocessing scanned manuscript images for handwriting recognition consists of layout analysis and text line extraction. In order to focus on handwriting recognition, we work directly with pre-segmented text line images in this paper. They were extracted with a semi-automatic procedure detailed in [5].

Next, the text foreground is determined by means of binarization. For the Saint Gall database, Sauvola's method is employed [4] and for the Parzival database, local edge enhancement is performed with Difference of Gaussians before applying a global threshold [5].

Finally, the binary images undergo a series of normalization operations in order to standardize their appearance before recognition. Normalization includes skew correction as well as a vertical division into an upper, middle, and lower region. An exemplary normalization result is shown in Figures $2 \mathrm{~b}$ and $3 \mathrm{~b}$. For further details, we refer to [5].

\subsection{Kanungo Noise}

The first degradation model was proposed by Kanungo et al. in [9] and was validated in [8]. The model distorts character edges by using a non-linear local selection process and a morphological closing operation. First, the selection process flips the value of some foreground and background pixels (foreground pixel changes to background and vice versa) according to a probability function. The probability function depends on a distance transform as follows:

- Foreground pixels: $p=\alpha_{0} e^{-\alpha d}+\eta$

- Background pixels: $p=\beta_{0} e^{-\beta d}+\eta$

where $d$ is the distance of a pixel to the nearest character edge. The two input parameters $\alpha$ and $\beta$ control the number of flipped pixels. The three parameters $\alpha_{0}, \beta_{0}$, and $\eta$ are considered as the initial inputs. Secondly, a morphological closing operation is applied to smooth the character edges. This model works well with binary images and is widely used to simulate the presence of noise for assessing the performances of different document analysis methods.

We consider three degradation levels with increasing distortion effect:

- Level 1: $\alpha=\beta=7, \alpha_{0}=\beta_{0}=1, \eta=0$

- Level 2: $\alpha=\beta=5.5, \alpha_{0}=\beta_{0}=1, \eta=0$

- Level 3: $\alpha=\beta=4.5, \alpha_{0}=\beta_{0}=1, \eta=0$

The effects of the different degradation levels are illustrated in Figures 2c and 3c. The number of flipped pixels ranges from 100-200 for level 1, 400-800 for level 2, and 800-1600 for level 3.

\subsection{Character Degradation}

The second degradation model is the character degradation model proposed in [10], which is specifically designed to 


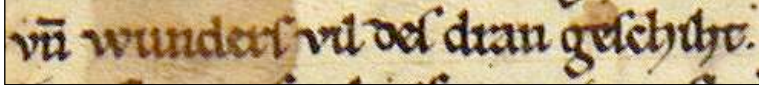

(a) Original

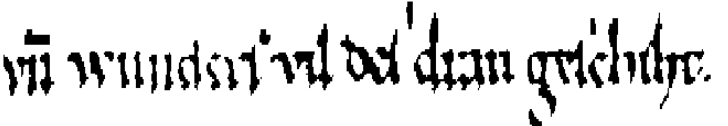

(b) Normalization

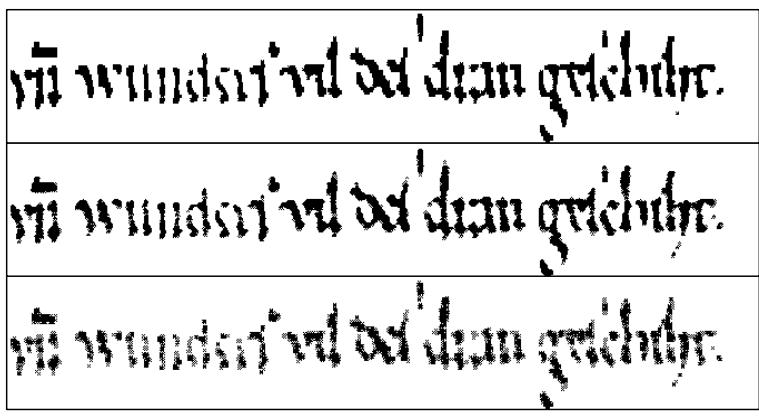

(c) Kanungo

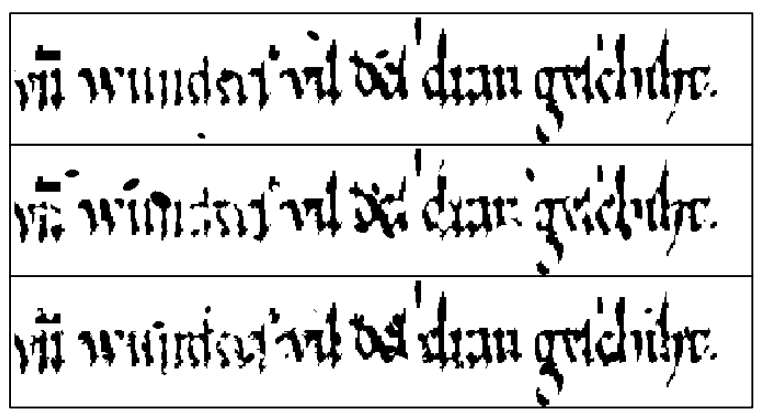

(d) Character

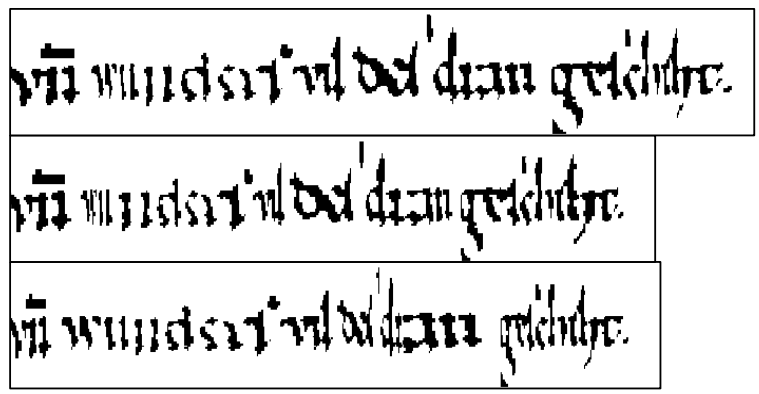

(e) Geometric

Figure 3: Image Degradation Parzival mimic typical noise regions in ancient documents such as ink splotches and white specks or streaks due to the age of the manuscripts. This kind of noise mostly appears in the neighborhood of the characters and can lead to touching and broken characters.

As detailed in [10], this noise can be simulated in three steps. First, the seed-points (centers of noise regions) are selected as the flipped pixels resulting from the non-linear local selection process proposed by Kanungo et al. [9]. Secondly, at each seed-point, a noise region is defined with three properties: shape, size, and direction. For example, an elliptic noise region has two axes (minor and major) which depend on the gradient vector $\vec{v}$ at its center and on two input parameters $a_{0}$ and $g$. The parameter $a_{0}$ controls the size of the elliptic region and $g$ its "flatness". The size parameter $a_{0}$ determines whether noise regions break, connect, or modify character strokes. Finally, in order to obtain degraded regions as realistic as possible, the gray value of the pixels inside each noise region is smoothed with a Gaussian function. Since we are working with binary images rather than grayscale images, this last step is omitted.

Again, we define three degradation levels with increasing distortion effect:

- Level 1: $\alpha=\beta=8.5, \alpha_{0}=\beta_{0}=1, \eta=0$ $g=0.6, a_{0}=5$

- Level 2: $\alpha=\beta=7, \alpha_{0}=\beta_{0}=1, \eta=0$ $g=0.6, a_{0} \in\{3,4, \ldots, 7\}$ (random selection)

- Level 3: $\alpha=\beta=7, \alpha_{0}=\beta_{0}=1, \eta=0$ $g=0.6, a_{0} \in\{3,4, \ldots, 10\}$ (random selection)

The effect is shown in Figures $2 \mathrm{~d}$ and $3 \mathrm{~d}$. The number of pixels in the elliptic noise regions ranges from 50-100 for level 1, 100-200 for level 2, and 200-400 for level 3.

\subsection{Geometric Distortion}

The third degradation model simulates a geometric surface deformation. It was proposed by J. Liang et al. in [13] to evaluate their restoration algorithm. First, this model maps every point in the plane of the original image to a point on a curved surface. The curved surface is then divided into into planar strips (e.g. quadrilaterals) that are mapped back to the image plane.

We employ this degradation model with different developable surfaces such as sinusoidal and parabolic. The distortion levels are controlled by the amplitude $a$ and wavelength $\lambda$ of the surface:

- Level 1: sinusoidal surface, $a=10, \lambda=0.5$

- Level 2: sinusoidal surface, $a=15, \lambda=0.25$

- Level 3: parabolic surface, $a=20, \lambda=0.2$

The effect is illustrated in Figures 2e and 3e. 


\section{RECOGNITION SYSTEM}

In this section, we briefly describe the hidden Markov model based recognizer used in the experiments. For a full description, we refer to $[16,6]$.

\subsection{Features}

Using a sliding analysis window with a width of 1 pixel moving from left to right, the binary text line images are represented by a sequence of feature vectors. Each window captures nine geometric features including the center of gravity, the second order moment, the fraction of black pixels, the contour positions, the deviation at the contours, and the number of black-white transitions. For more details, we refer to $[16]$.

\subsection{Training}

Morphological character models consist of a series of states arranged in a linear topology. Each state captures the distribution of the sliding window features with a mixture of Gaussians with diagonal covariance matrices. Training is performed with the Baum-Welch algorithm [19] based on known transcriptions of text line images.

We employ a training procedure that increases the number of Gaussians $G$ incrementally. Starting with $G=1$, the training set is processed $I$ times in the first training epoch. Then, the number of Gaussians is incremented to $G=2$ by splitting and the training set is again processed $I$ times in this second training epoch. This procedure is continued until a maximum number of Gaussians is reached.

\subsection{Recognition}

Two goal functions are optimized during recognition with the Viterbi algorithm [19]. First, the best match of the image with the trained morphological character models is established. Secondly, the most likely sequence of words is found with respect to a bigram language model that describes the probability of a word given the preceding word. The two goal functions are balanced with a grammar scale factor and a word insertion penalty [24].

We estimate the word bigrams on all training and validation text lines and smooth them with the modified Kneser-Ney method [11] to cope with unseen word bigrams. Furthermore, we use a closed vocabulary that contains all words of the database, even those of the test set. It is a common practice to establish comparable results without out-ofvocabulary words. For the Saint Gall database, the recognition vocabulary contains 5, 762 words and for the Parzival database, the vocabulary contains 4,934 words.

\section{EXPERIMENTAL EVALUATION}

We have conducted an experimental evaluation of the proposed image degradation models on the Saint Gall and Parzival data sets (see Section 2).

All three models, namely Kanungo noise, character degradation, and geometric distortion, are applied to the training set individually to create one distorted version of each learning sample. In effect, the size of the training set is doubled. It is used to train a new recognition system, which is then
Table 2: Database Setup

\begin{tabular}{lcc}
\hline & Saint Gall & Parzival \\
\hline Training & 468 & 2237 \\
Validation & 235 & 912 \\
Test & 707 & 1328 \\
\hline
\end{tabular}

Table 3: Recognition Accuracy

\begin{tabular}{lcc}
\hline & Saint Gall & Parzival \\
\hline Reference & 88.99 & 83.89 \\
Kanungo & $90.15+1.16$ & $86.95+3.06$ \\
Character & $90.42+1.43$ & $85.37+1.48$ \\
Geometric & $89.25+0.26$ & $85.66+1.77$ \\
Combined & $90.81+1.82$ & $87.12+3.23$ \\
\hline
\end{tabular}

compared with the reference system trained on the original training set.

In addition to an individual model evaluation, we also combine up to two noise models by adding their degradation images to the same training set, which is then three times larger than the original training set.

\subsection{Setup}

First, the text lines of both data sets are split into disjoint sets for training the HMM, validation of system parameters, and testing the final performance. The number of text lines are indicated in Table 2 for each set.

For the image degradation models, the best degradation level $L \in\{1,2,3\}$ is determined on the validation set. Also, the best pair of degradation models for the model combination is found with respect to the validation accuracy.

System parameters of the HMM recognizer, which are optimized on the validation set, include the number of training iterations $I \in\{2,3,4,5\}$ per training epoch and the final number of Gaussian mixtures $G \in\{5,10, \ldots, 30\}$. The number of HMM states and the language model parameters have been adopted from previous experiments [23].

\subsection{Results}

The word accuracy results are indicated in Table 3 for each individual image degradation model as well as for the best combination of two degradation models. Next to the word accuracy, the improvement over the reference system is indicated. With the exception of geometric distortions on the Saint Gall database, 7 out of 8 improvements over the reference system are statistically significant (t-test, $\alpha=0.05$ ).

Table 4 lists the relative word error reduction for all models. The reductions are also shown in Figure 4. The synthetic learning samples realize an error reduction of up to $16.53 \%$ on the Saint Gall database, achieving an accuracy of $90.81 \%$. On the Parzival database, an error reduction of up to $20.05 \%$ is achieved and an accuracy of $87.12 \%$ is reported. These re- 
Table 4: Error Reduction

\begin{tabular}{lcc}
\hline & Saint Gall & Parzival \\
\hline Kanungo & 10.54 & 18.99 \\
Character & 12.99 & 09.19 \\
Geometric & 02.36 & 10.99 \\
Combined & 16.53 & 20.05 \\
\hline
\end{tabular}

Table 5: Degradation Levels

\begin{tabular}{lcc}
\hline & Saint Gall & Parzival \\
\hline Kanungo & 1 & 1 \\
Character & 2 & 1 \\
Geometric & 1 & 3 \\
\hline
\end{tabular}

sults clearly demonstrate the benefit of generating synthetic learning samples using image degradation.

When comparing the individual models, the character degradation model and the Kanungo noise tend to outperform the geometric distortion. Table 5 lists the optimal degradation levels of the different models. In 4 out of 6 cases, the lowest degradation level was pefered.

For both data sets, the combination of noise models achieves the best result. For the Saint Gall database, the optimal pair of models was (Character, Geometric) and for the Parzival database, the optimal pair was (Kanungo, Character). The combination results demonstrate that multiple sources of degradations are desirable for the synthetic generation of learning samples.

\section{CONCLUSIONS}

In this paper, we have investigated image degradation models to generate synthetic learning samples for historical handwriting recognition. The three proposed degradation models include Kanungo noise, character degradation, and geometric distortion. They have been applied to distort binary text line images for training a hidden Markov model based recognizer.

On the medieval Saint Gall and Parzival data sets, significant error reductions could be achieved with the synthetic learning samples. For closed-vocabulary recognition, the best word accuracy results were $90.81 \%$ for the Saint Gall database and $87.12 \%$ for the Parzival database. This corresponds to an error reduction over the baseline system of $16.53 \%$ and $20.05 \%$, respectively. For both data sets, the best results were achieved by a combination of different degradation models.

In the context of historical manuscripts, the importance of learning samples cannot be overemphasized. Especially when bootstrapping a new handwriting recognition system for a new kind of historic script, every learning sample counts. Image degradations have shown a promising potential to incorporate more variability in the training set, which leads

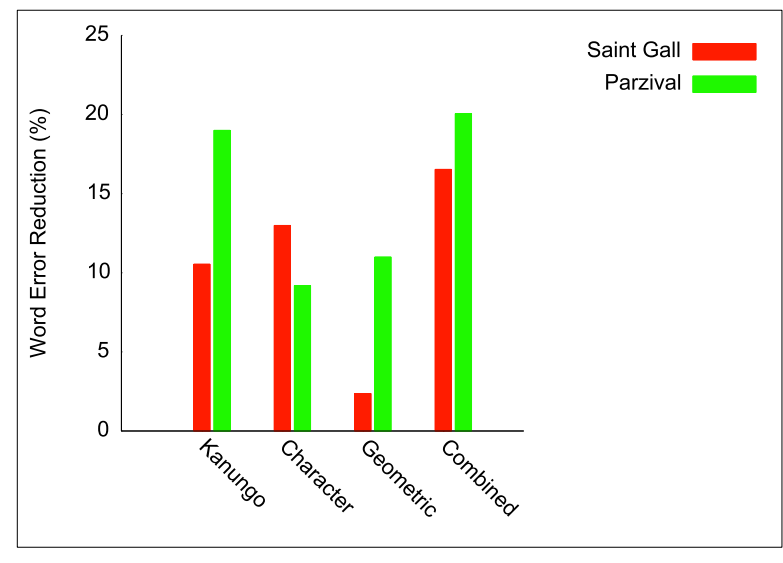

Figure 4: Error Reduction.

to a better generalization capability of the recognizer.

Future work includes improvements in the degradation models, in particular the investigation of realistic 3-dimensional distortions of the writing support. The goal is to create realistic manuscript images that incorporate several types of degradations.

\section{ACKNOWLEDGMENTS}

This work has been supported by the Swiss National Science Foundation fellowship project PBBEP2_141453.

\section{REFERENCES}

[1] A. Antonacopoulos and A. Downton. Special issue on the analysis of historical documents. Int. Journal on Document Analysis and Recognition, 9(2):75-77, 2007.

[2] H. S. Baird. The State of the Art of Document Image Degradation Modeling. In In Proc. of 4 th IAPR International Workshop on Document Analysis Systems, Rio de Janeiro, pages 1-16, Rio de Janeiro, Brazil, 2000.

[3] M. Delalandre, E. Valveny, T. Pridmore, and D. Karatzas. Generation of Synthetic Documents for Performance Evaluation of Symbol Recognition \& Spotting Systems. Int. J. Doc. Anal. Recognit., 13(3):187-207, Sept. 2010.

[4] A. Fischer, V. Frinken, A. Fornés, and H. Bunke. Transcription alignment of latin manuscripts using hidden Markov models. In Proc. 1st Int. Workshop on Historical Document Imaging and Processing, pages 29-36, 2011.

[5] A. Fischer, E. Indermühle, H. Bunke, G. Viehhauser, and M. Stolz. Ground truth creation for handwriting recognition in historical documents. In Proc. 9th Int. Workshop on Document Analysis Systems, pages 3-10, 2010.

[6] A. Fischer, A. Keller, V. Frinken, and H. Bunke. Lexicon-free handwritten word spotting using character HMMs. Pattern Recognition Letters, 33(7):934-942, 2012.

[7] D. D. Jian Zhai, Liu Wenyin and Q. Li. A Line Drawings Degradation Model for Performance Characterization. In Proc. 7th ICDAR, pages 
1020-1024, Edinburgh, Scotland, August 2003.

[8] T. Kanungo, R. Haralick, H. Baird, W. Stuezle, and D. Madigan. A statistical, Nonparametric Methodology for Document Degradation Model Validation. IEEE Trans. Pattern Anal. Mach. Intell., 22(11):1209 - 1223, 2000.

[9] T. Kanungo, R. M. Haralick, and I. Phillips. Global and Local Document Degradation Models. In Proc. of the ICDAR, pages 730-734, Tsukuba Science City, Japan, Oct. 1993.

[10] V. Kieu, M. Visani, N. Journet, J. P. Domenger, and R. Mullot. A Character Degradation Model for Grayscale Ancient Document Images. In Proc. of the $I C P R$, pages 685-688, Tsukuba Science City, Japan, Nov. 2012.

[11] R. Kneser and H. Ney. Improved backing-off for m-gram language modeling. In Proc. Int. Conf. on Acoustics, Speech, and Signal Processing, pages 181-184, 1995.

[12] A. L. Koerich, R. Sabourin, and C. Suen. Large vocabulary off-line handwriting recognition: A survey. Pattern Analysis and Applications, 6:97-121, 2003.

[13] J. Liang, D. DeMenthon, and D. S. Doermann. Geometric Rectification of Camera-Captured Document Images. IEEE Trans. Pattern Anal. Mach. Intell., 30(4):591-605, 2008.

[14] L. Likforman-Sulem, A. Zahour, and B. Taconet. Text line segmentation of historical documents: A survey. Int. Journal on Document Analysis and Recognition, 9(2):123-138, 2007.

[15] R. Loce and W. Lama. Halftone Banding due to Vibrations in A Xerographic Image Bar Printer. Journal of Imaging Technology, 16(1):6-11, 1990.

[16] U.-V. Marti and H. Bunke. Using a statistical language model to improve the performance of an HMM-based cursive handwriting recognition system. Int. Journal of Pattern Recognition and Artificial Intelligence, 15:65-90, 2001.

[17] C. M. Moghaddam R.F. Low Quality Document Image Modeling and Enhancement. Int. Journal on Document Analysis and Recognition, 11(4):183-201, 2009.

[18] M. Mori, A. Suzuki, A. Shio, and S. Ohtsuka. Generating New Samples from Handwritten Numerals Based on Point Correspondence. In Proc. 7th Int. Workshop on Frontiers in Handwriting Recognition, pages 281-290, Amsterdam, Netherlands, 2000.

[19] L. Rabiner. A tutorial on hidden Markov models and selected applications in speech recognition. Proceedings of the IEEE, 77(2):257-285, 1989.

[20] T. Varga and H. Bunke. Effects of Training Set Expansion in Handwriting Recognition Using Synthetic Data. In Proc. 11th Conf. of the Int. Graphonomics Society, pages 200-203, Scottsdale, AZ USA, Nov. 2003. Citeseer.

[21] T. Varga and H. Bunke. Generation of Synthetic Training Data for an HMM-based Handwriting Recognition System. In Proc. 7th ICDAR, pages 618-622, Edinburgh, Scotland, August 2003.

[22] M. Visani, V. Kieu, A. Fornés, and N. Journet. The ICDAR/GREC 2013 Music Scores Competition on Staff Removal. In Accepted for publication in
Document Analysis and Recognition (ICDAR), 2013 International Conference on, 2013.

[23] M. Wüthrich, M. Liwicki, A. Fischer, E. Indermühle, H. Bunke, G. Viehhauser, and M. Stolz. Language model integration for the recognition of handwritten medieval documents. In Proc. 10th Int. Conf. on Document Analysis and Recognition, volume 1, pages 211-215, 2009.

[24] M. Zimmermann and H. Bunke. N-gram language models for offline handwritten text recognition. In Proc. 9th Int. Workshop on Frontiers in Handwriting Recognition, pages 203-208, 2004. 